\title{
IT-Based Self-Monitoring Interventions to Promote Physical Activity and Weight Loss: A Meta-Analysis of Change-from-Baseline Effects
}

\author{
Jinglu Jiang \\ HEC Montréal \\ jinglu.jiang@hec.ca
}

\author{
Ann-Frances Cameron \\ HEC Montréal \\ ann-frances.cameron@hec.ca
}

\begin{abstract}
IT-based self-monitoring (ITSM) has attracted increasing interest as a strategy for chronic disease self-management and day-to-day fitness promotion. Despite the increasing popularity of various selfmonitoring technologies such as fitness trackers and biosensors, their effectiveness is less certain. The objective of the current review is to determine the effectiveness of ITSM interventions on two types of key chronic care outcomes: weight management and physical activity. A systematic review employing a meta-analysis identified 42 ITSM studies that report change-from-baseline effects on weight and physical activity-related outcomes. Overall, a small effect size is found for body weight, BMI, waist circumferences, and step-based physical activity. The effect estimates on time-based physical activity are moderate. However, the effects on physical activity show variability and potential publication bias. A post-hoc analysis of the effects of ITSM on self-efficacy exhibit a small yet significant effect size, which shows the potential mediating role of patients' psychological outcomes on the ultimate behavioral outcomes. In summary, ITSM is a potentially useful approach to manage weight and physical activity. Further study is needed to determine the source of heterogeneity as well as the types of ITSM interventions that are effective for weight, physical activity, but also other chronic care outcomes.
\end{abstract}

\section{Introduction}

According to the WHO, six of the top ten global causes of death are chronic diseases [1]. Physical inactivity and being overweight are the two leading risk factors associated with a wide range of chronic diseases such as diabetes, cardiovascular diseases, asthma, depression, osteoarthritis pain and several cancers [2] [3] [4]. Therefore, improving weight management and facilitating physical activity (PA) are important in managing these chronic conditions.
Traditional management of weight and PA involves paper-based self-recording and frequent clinic measurement visits that may generate heavy burdens on the healthcare system [5]. With the popularity of new eHealth technologies (e.g. smart devices, fitness trackers and virtual reality for healthcare purposes), a new paradigm has emerged, focusing on an individual's self-monitoring (SM) of chronic conditions using IT. Patients use systematic approaches to longitudinally monitor and manage their chronic conditions in digital ways, which increasingly moves the responsibility of chronic care from the medical clinic to the home [6] [7] [8]. Of the various IT-based SM programs, the most widely implemented are those focused on weight management and physical activity. These ITSM programs are not only for specific groups of patients, but also for society as a whole [9]. Thus, understanding the effectiveness of ITSM on weight management and physical activity has meaningful practical significance.

The concept of ITSM seems self-evident, so many studies do not define it clearly. However, the use of this term is confusing, and in many cases, ITSM is interchangeable with other broader methods such as self-management and self-care, and narrower techniques such as self-tracking and self-recording. The concept analysis studies by [10] and [11] show that SM should include two attributes: (1) selfawareness of bodily symptoms and conditions, and (2) self-recording of the observations and measurements. When SM of weight and PA is performed for chronic care, IT simplifies data recording and can potentially improve patients' awareness, interpretation and appraisal of their conditions with digital features such as personalized feedback, real-time dashboards and social networking. However, these digital approaches also bring new challenges. Patients and their families may need to make extra efforts to persist selfregulation. They need to be trained to properly prepare the IT systems, faithfully record data, and correctly analyze the information to support proactive decision making and meaningful care [12]. Consequently, ITSM 
often encounters issues such as intermittent nonadherence and invalid evidence generation [13] [14].

Since the SM approach has been documented as a key strategy for chronic disease self-management, and various empirical investigations have focused on the delivery of weight management and physical activityrelated SM for various chronic conditions, it is essential to understand the effectiveness of such interventions. A synthesis of these findings helps us understand the effectiveness of weight and physical activity ITSM, and provides useful directions for future research.

The aim of the present review is to assess the effectiveness of IT-based SM interventions for weight management and physical activity, both of which are important for managing chronic disease. We focus on physical outcomes (i.e. improvement in body weight and PA level) to evaluate effectiveness, and post-hoc analysis of one psychological outcome that emerged (i.e. improvement in self-efficacy). By quantitatively synthesizing the effect size, useful insights are generated by combining the results of comparable studies and identifying the potential issues in effectively delivering ITSM interventions.

\section{Background and literature review}

ITSM is a frequently used technique in cognitivebehavioral therapies and behavioral change interventions to promote health outcomes [23] [24]. However, in healthcare literature, the focus is on the effectiveness of a treatment (or an intervention) as a bundle, which may or may not involve IT components, whereas the role of the IT-based component is of less interest. Since IT-supported treatment delivery has become increasingly common in recent years, and has been addressed in various treatment studies in both the healthcare and IS fields, it is an opportune moment to examine the usefulness of these ITSM-based interventions.

We have identified five relevant systematic literature reviews and meta-analyses. Burke et al. [15] systematically reviewed 22 studies on weight loss SM published between 1993 and 2009, predominantly employing paper-based SM. George et al. [16] qualitatively synthesized the effectiveness of intervention delivery modes in 14 studies published between 1990 and 2010 on physical activity interventions for adult males. Greaves et al. [17] provided an umbrella review of 30 existing reviews published between 1998 and 2008 on physical activity intervention for diabetes, qualitatively synthesizing key intervention components. Olander et al. [18] provided a meta-analysis of effective behavioral change techniques in promoting obese individuals' physical activity based on 42 articles, finding a medium effect size. Van den Berg et al. [19] also qualitatively synthesized 10 studies on Internet-based physical activity interventions conducted up until 2006.

Whereas prior syntheses have provided insightful evidence regarding the useful techniques (or intervention components) of weight management and physical activity, a comprehensive quantitative synthesis that includes more recent SM technologies and goes beyond one type of disease and single gender is necessary to understand the recent development in the field. Accordingly, this study specifically focuses on ITSM, which is more relevant to the current trend of chronic disease self-care practices. The meta-analytic synthesis of the intervention effectiveness based on recent empirical studies (published between 2006 and 2016) also contributes to evidence-based healthcare practices.

\section{Methodology}

\subsection{Inclusion and exclusion criteria}

We followed a formal meta-analysis literature review process [33] and systematically searched eight databases (i.e. EBSCO host [including MEDLINE], ABI/INFORM, ACM digital library, ScienceDirect, IEEE Xplore, JSTOR, PsycINFO and Web of Knowledge). Since our target was studies using ITbased SM intervention for weight management and physical activity in chronic disease management, articles published in peer-reviewed journals and conference proceedings from 2006 to 2016 were searched using the keywords "self-monitor*", "selfsurveillance", "personal analytics", "self-track*", "personal informatics", and "electronic personal archive". Articles were screened by titles and abstracts, with the research team reading the full text when needed. Eligible studies met the following criteria:

- Study is empirical and uses an experimental approach (i.e. randomized controlled trial, non-randomized controlled trial, quasiexperiment and pretest-posttest design);

- Study examines a specific chronic disease, or the management or control of high-risk behaviors that relate to chronic disease;

- The intervention or experiment design includes IT-based SM components;

- Weight-related and PA-related outcomes are directly examined.

Studies were excluded if they: (1) are nonempirical, (2) are not related to any chronic disease or high-risk factors, (3) are non-technological and do not involve IT-based SM, (4) involve no weight 
management or physical activity outcomes, (5) use an incompatible definition of SM (e.g. SM as a personality trait that focuses on individuals' control of their expressive behavior according to social cues), (6) only focus on technical development or measurement development, (7) do not involve human data, and (8) only provide descriptive statistics without further investigating the relationships.

The database search yielded an initial 2,985 studies. After removing the duplicates, systematically applying the screening criteria and performing forward and backward searches [25], 42 studies are eligible for further analysis. Figure 1 presents the articles excluded in each step.

The effectiveness of an intervention can be assessed by comparing 1) posttest to the baseline condition, or 2) treatment group(s) to the control group. This study does not include the latter type because we found that the experiment designs are quite diverse and study results are difficult to meaningfully compare. The majority of them used alternative treatment as the control (e.g., weight loss SM intervention vs. weight loss coaching intervention [30] [31]) rather than using a true control, waitlist or placebo, making it less meaningful to compare the between-group effects among the studies. For the first type, studies employing the change-from-baseline effect use the baseline condition as the experiment group's own control, which is an efficient and valid way to remove between-group variability [20]. The downside of this approach is that when the measurement of outcomes is unstable, the measurement error may be larger than the betweengroup variability. However, in our case, weight and physical activity measures can be precisely captured and are quite stable.

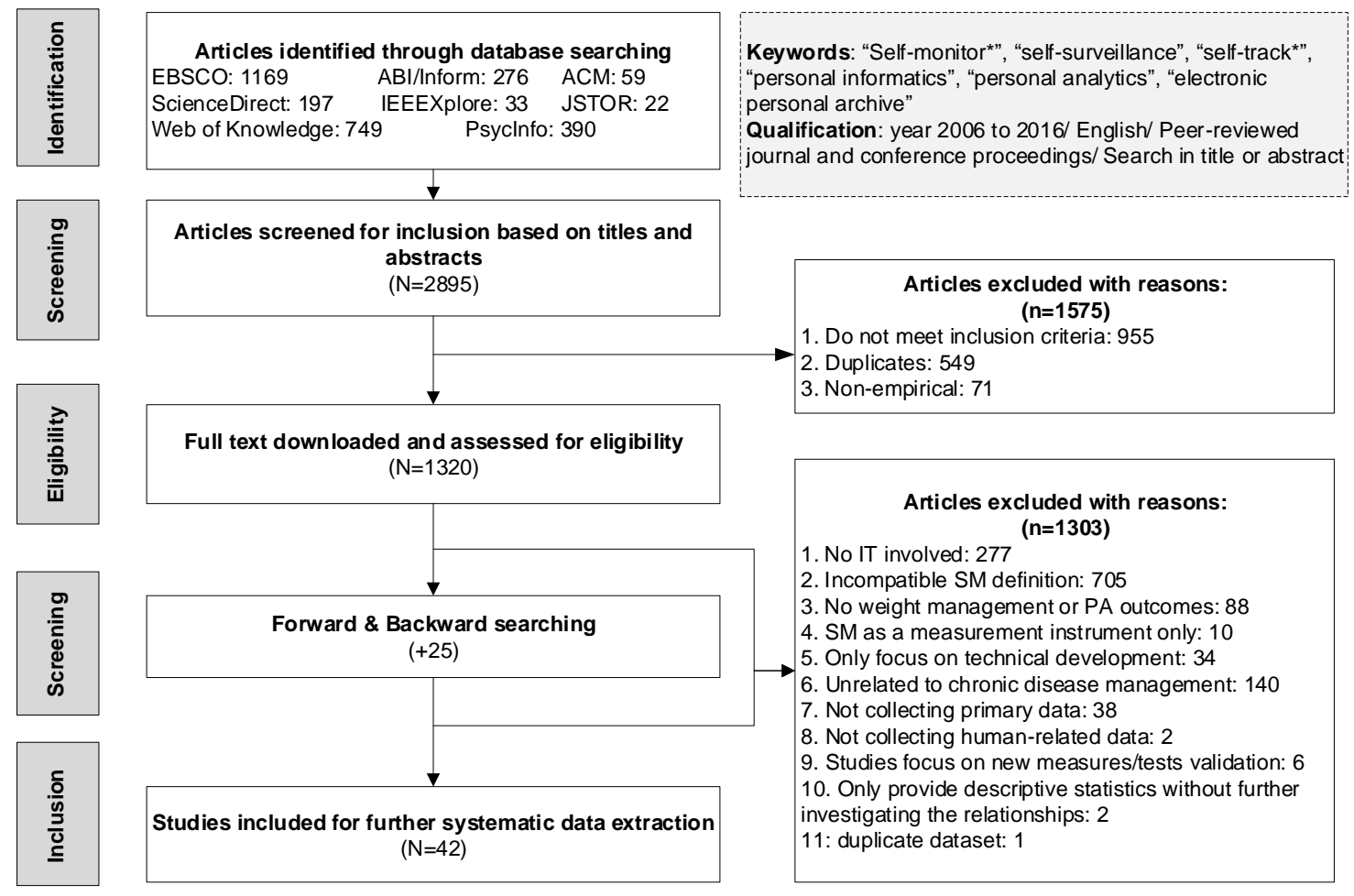

Note. 12 studies were not included in the final analysis due to lack of appropriate information for effect size calculation.

\section{Figure 1. Flow chart describing the number of studies retrieved and screening process}

\subsection{Data extraction and analysis}

Descriptive information, participant characteristics and intervention information were extracted for each study. We also extracted baseline and posttest sample sizes, means and standard deviations (SD). For studies with multiple interventions, we extracted the abovementioned statistics for each intervention using
ITSM. Since these interventions are independent from each other, the effect is counted independently as well.

We calculated standardized mean differences for each eligible intervention to measure effect size [19]. Adjusted Hedges' $g$ was used in our calculation, which uses Bessel's correction to estimate the mean differences in units of the pooled standard deviation [26]. Hedges' $g$ is very similar to Cohen's $d$ (i.e. 
another frequently used effect size measure). While they perform similarly when the sample size is large, Hedges' $g$ outperforms Cohen's $d$ when the sample size is small (i.e. $N$ <20) [32], which is the case for many of our studies. Note that although the common suggested effect size thresholds are 0.2 (small), 0.5 (moderate) and 0.8 (large) [27], these numbers are not absolute cutoffs but relative to each other and the research field. For the studies that did not report SD, we imputed missing SD from confidence intervals, standard errors, $t$ values, $P$ values and $F$ values if available [20]. If none of these statistics were available, we contacted the authors for further information. Currently 12 studies are not included in our analysis due to missing SD; however, they will be included once the missing data is received.

Heterogeneity, which shows the variation of study outcomes, is represented by $I^{2}$ statistic, which is derived from Cochran's $Q$ (threshold: below $40 \%$ as small and above $75 \%$ as high) [20]. This statistic indicates the inconsistency of the results for the set of studies. Therefore, meta-analytic results with high heterogeneity may indicate that the results do not uniformly represent the set of studies and may suggest the existence of subgroups or moderator effects.

In addition to two measurement points for the baseline and posttest, three studies reported additional measurement points during the intervention, and five studies reported additional follow-up measurements. For the main body of our analysis, for the posttest measurement point, we used the first measurement taken after the end of intervention in order to enhance the comparability of the studies' results.

\section{Results}

\subsection{Study characteristics}

In the following sections, we present the analysis of the 30 studies for which we are able to calculate effect sizes. Descriptive characteristics are presented in Table 1 below. For the type of IT that supports SM, web and pedometer were the most frequently used means, which is a shift from the popularity of paper and PDA approaches ten years ago [15]. For the studies that reported the race of participants, white people were dominant, and a majority of the studies investigated female health issues. It should also be noted that ITSM was usually accompanied by other complementary components such as goal setting, feedback and education sessions. More recent trends also show the potential of incorporating digital social networking into a healthcare intervention. The majority of interventions had a duration of three to six months.

\subsection{Effects on weight management}

Table 2 shows the impact of IT-based SM interventions on various outcomes of interest. The

Table 1. Descriptive data for the studies

\begin{tabular}{|c|c|c|}
\hline & Category & \# of studies \\
\hline \multirow[t]{6}{*}{ IT type } & PDA & 2 \\
\hline & Computer & 2 \\
\hline & Web & 13 \\
\hline & Mobile & 9 \\
\hline & Wearable & 4 \\
\hline & Pedometer & 10 \\
\hline \multirow{5}{*}{$\begin{array}{l}\text { Patient- } \\
\text { Race }\end{array}$} & $>60 \%$ white & 13 \\
\hline & $>60 \%$ black & 3 \\
\hline & $>60 \%$ Hispanic & 1 \\
\hline & mixed & 4 \\
\hline & Unclear & 10 \\
\hline \multirow[t]{5}{*}{ Patient- Sex } & all female & 5 \\
\hline & $<=50 \%$ male & 21 \\
\hline & $>50 \%$ male & 3 \\
\hline & all male & 1 \\
\hline & Unclear & 1 \\
\hline \multirow{6}{*}{$\begin{array}{l}\text { Patient- } \\
\text { average age }\end{array}$} & below 30 & 2 \\
\hline & $31-40$ & 2 \\
\hline & $41-50$ & 18 \\
\hline & $51-60$ & 6 \\
\hline & $61-70$ & 5 \\
\hline & Unclear & 3 \\
\hline \multirow{4}{*}{$\begin{array}{l}\text { Intervention- } \\
\text { components }\end{array}$} & Goal setting/assignment & 16 \\
\hline & feedback & 15 \\
\hline & education/counselling & 16 \\
\hline & social & 7 \\
\hline \multirow{3}{*}{$\begin{array}{l}\text { Intervention- } \\
\text { duration }\end{array}$} & $<=3$ months & 18 \\
\hline & $<=6$ months & 10 \\
\hline & $>6$ months & 2 \\
\hline \multirow{4}{*}{$\begin{array}{l}\text { Outcome } \\
\text { type }\end{array}$} & weight related & 19 \\
\hline & PA related & 15 \\
\hline & Self-efficacy related & 7 \\
\hline & both weight \& PA & 7 \\
\hline
\end{tabular}

Note. 30 studies in total and 41 independent interventions.

effect size of weight management related outcomes (i.e. body weight, BMI and waist circumference) is presented in figures 2, 3 and 4 . The forest plot lists the subgroup of studies for each outcome on the left-hand side, along with their weights based on sample size and the effect size (i.e. standardized mean differences) for each pair of comparisons (i.e. posttest vs. baseline). A standardized mean difference favoring baseline means that the baseline score is greater than the posttest score (indicating, for example, weight loss). The diamond at the bottom is the meta-analyzed effect size (i.e. weighted effect size for the whole set of studies). 
Heterogeneity tests and the effect size significance test are listed at the bottom. These detailed figures can be used by future researchers who wish to quickly determine which results were found in which studies.

Table 2. Effect size summary with $95 \% \mathrm{Cl}$

\begin{tabular}{|c|c|c|c|c|c|c|}
\hline Outcome & $\begin{array}{c}\text { Body weight } \\
(\mathrm{kg})\end{array}$ & BMI & $\begin{array}{c}\text { Waist } \\
\text { circumference }\end{array}$ & $\begin{array}{c}\text { time-related } \\
\text { physical activity }\end{array}$ & $\begin{array}{c}\text { step-related } \\
\text { physical activity }\end{array}$ & Self-efficacy \\
\hline \# of studies & 12 & 12 & 5 & 8 & 6 & 6 \\
\hline \# of interventions & 16 & 18 & 7 & 8 & 7 & 7 \\
\hline Total participants & 1255 & 1710 & 495 & 513 & 381 & 397 \\
\hline $\begin{array}{r}\text { Effect estimate } \\
(\mathrm{ES}, 95 \% \mathrm{Cl})\end{array}$ & $\begin{array}{c}0.31 \\
{[0.20,0.42]} \\
\text { (small) } \\
\end{array}$ & $\begin{array}{c}0.15 \\
{[0.06,0.25]} \\
\text { (small-negligible) }\end{array}$ & $\begin{array}{c}0.30 \\
{[0.12,0.48]} \\
(\text { small })\end{array}$ & $\begin{array}{c}-0.63 \\
{[-0.81,-0.46]} \\
\text { (moderate) }\end{array}$ & $\begin{array}{c}-0.34 \\
{[-0.55,-0.13]} \\
(\text { small }) \\
\end{array}$ & $\begin{array}{c}-0.39 \\
{[-0.59,-0.19]} \\
\text { (small) }\end{array}$ \\
\hline Heterogeneity $\left(\mathrm{I}^{2}\right)$ & $0 \%$ [low] & $0 \%$ [low] & $0 \%$ [low] & $56 \%$ [moderate] & $88 \%$ [high] & $18 \%$ [small] \\
\hline
\end{tabular}

In general, the meta-analysis shows small but significant effect sizes for all three types of weight outcomes. Sixteen change-from-baseline comparisons are included in body weight change analysis using the fixed effect model (due to the homogenous nature of the studies based on small $\mathrm{I}^{2}$ ), which gives an effect size of 0.31 (95\% CI $0.2-0.42$, $\mathrm{p}<0.001$ ). Similarly, the effect size estimates are 0.3 (95\% CI 0.12-0.48, $\mathrm{p}=0.001)$ and 0.15 (95\% CI 0.06$0.25, \mathrm{p}=0.002$ ) for waist circumferences and BMI change respectively.

Publication bias is presented in the funnel plots in Appendix A, which compares standard error of the effect against standard mean deviation. A plot resembling a symmetrical funnel indicates the absence of publication bias. Visual inspection of the funnel plots for weight-related studies finds symmetrical patterns for BMI, but less symmetry for body weight, indicating potential publication bias (see Appendix A.1 \& A.2). The source of bias varies (e.g. poor study design, inadequate analysis, biased sample, etc.), and future research needs to formally assess the risk of bias [28].

\subsection{Effects on physical activity}

Figures 5 and 6 show the effect of ITSM interventions on physical activity measured by timebased or step-based indicators. Results show a significant moderate effect size of -0.63 (95\% CI [$0.81,-0.46], \mathrm{p}<0.001)$ on time-based outcomes and a significant small effect size of -0.34 (95\% CI [-0.55,

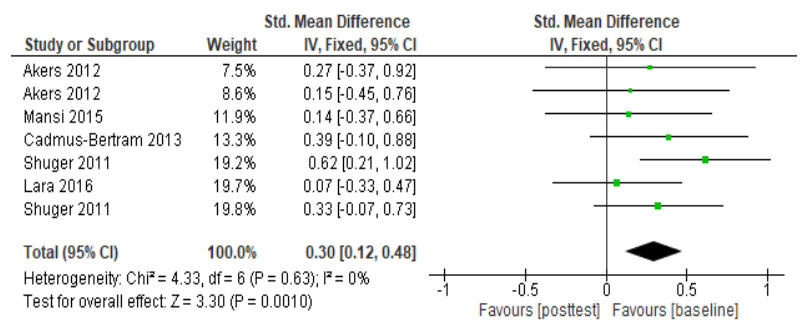

Figure 2. Forest plot showing waist circumference effect size with $95 \% \mathrm{Cl}$

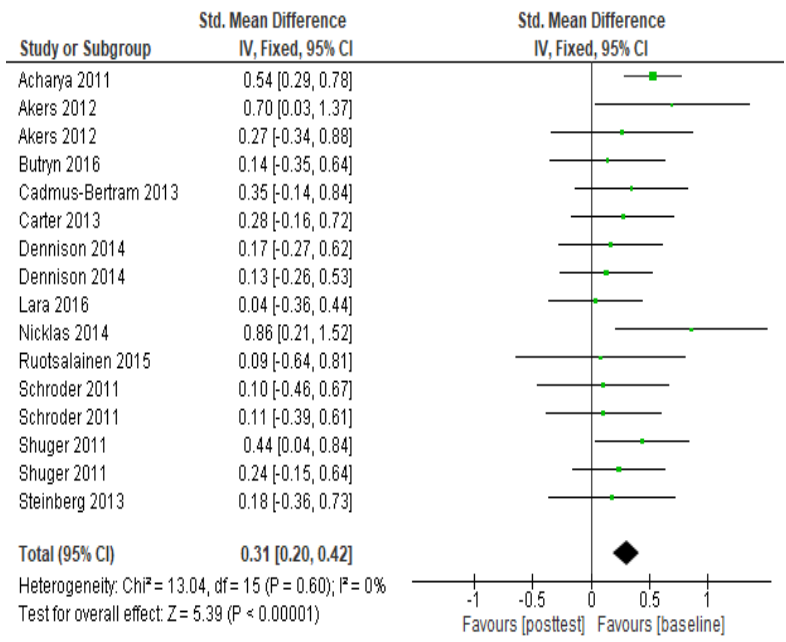

Figure 3. Forest plot showing body weight effect size with $95 \% \mathrm{Cl}$ for each intervention

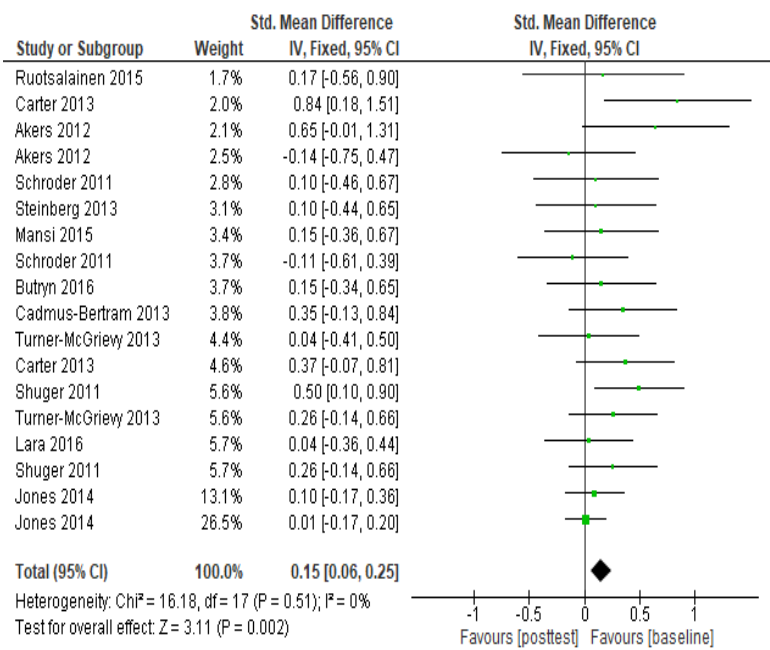

Figure 4. Forest plot showing BMI effect size with $95 \% \mathrm{Cl}$ for each intervention 
-0.13], $\mathrm{p}=0.001$ ) on step-based outcomes. The negative value indicates posttest physical activity is greater than the baseline. The magnitude of the metaanalyzed effect size implies the effectiveness of ITSM interventions, which is comparable to previous meta-analysis of how behavior change techniques influence physical activity $(d=0.5$ in [18]).

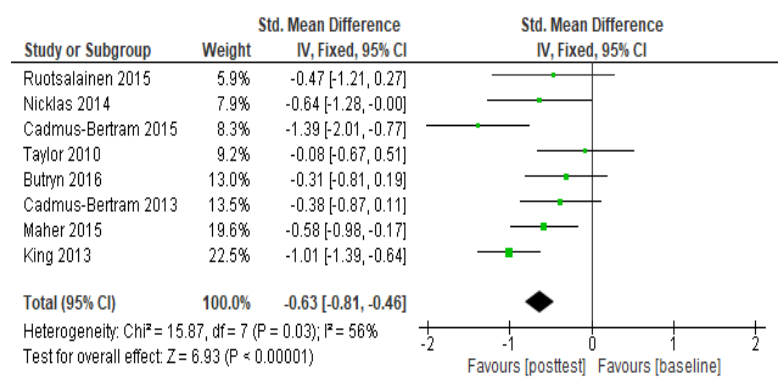

Figure 5. Forest plot showing time-based PA effect size with $95 \% \mathrm{Cl}$ for each intervention

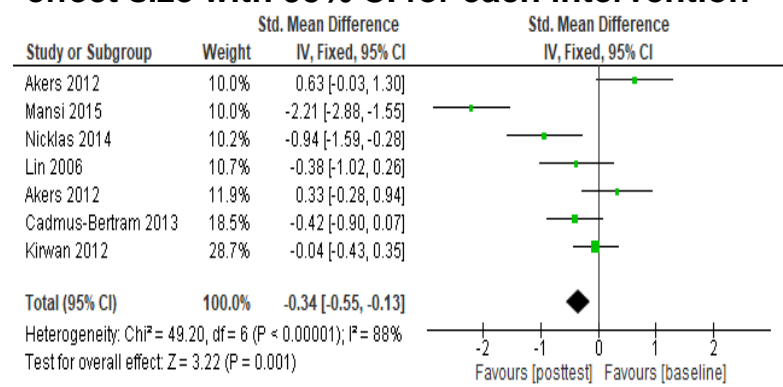

Figure 6. Forest plot showing step-based PA effect size with $95 \% \mathrm{Cl}$ for each intervention

Since physical activity measures varied, we further subgroup the measurements to see if there are potential variations on the effect size. For time-based measures, if only moderate PA time is taken into consideration as the outcome, it gives a significant moderate effect size of -0.7 (95\% CI [-1.2, -0.2], $\mathrm{p}=0.006$ ), which is comparable to the main analysis that takes the average of similar measures as the outcome (e.g. average the overall exercise time and moderate exercise time). For step-based measures, one outlier study found a significant reduction in PA steps (i.e. Akers 2012, 2 interventions). If we remove this one outlier, it gives an even stronger significant moderate effect size of -0.57 (95\% CI [-0.81, -0.34], $\mathrm{p}<0.001)$. A closer examination of this outlier study found that the intervention focused on water consumption and diet, meaning that physical activity is a secondary outcome with did not receive much emphasis in the study design, thereby the participants may not have taken the step-tracking as seriously as the other components of the intervention.

Heterogeneity of physical activity results are relatively high for both outcomes, which may be due to the combination of different measures and non- comparable intervention designs. This suggests that the results do not uniformly represent the set of studies and may suggest the existence of subgroups or moderator effects. The funnel plots in Appendix A.4 and A.5 also show potential publication bias.

\subsection{Effects on self-efficacy}

During our analysis, self-efficacy was reported in six studies and emerged as a key potential contributor to weight management and/or physical activity. Thus, we also conduct post-hoc analysis of the impacts on self-efficacy, hoping to shed light on the potential source of heterogeneity and understand how ITSM produces effects on weight management and PA.

An individuals' belief in their ability to perform a specific behavior is often theorized as an important antecedent or mediator of the designated behaviors [21] [22]. Thus, the effect of ITSM intervention on participants' self-efficacy may help explain the effectiveness of an intervention on the ultimate weight and physical activity outcomes. As shown in Figure 7, the effect size estimate is small-to-moderate $(-0.39,95 \%$ CI $[-0.59,-0.19], \mathrm{p}<0.001)$ with small heterogeneity of $18 \%$, indicating relatively consistent study effects on self-efficacy improvement.

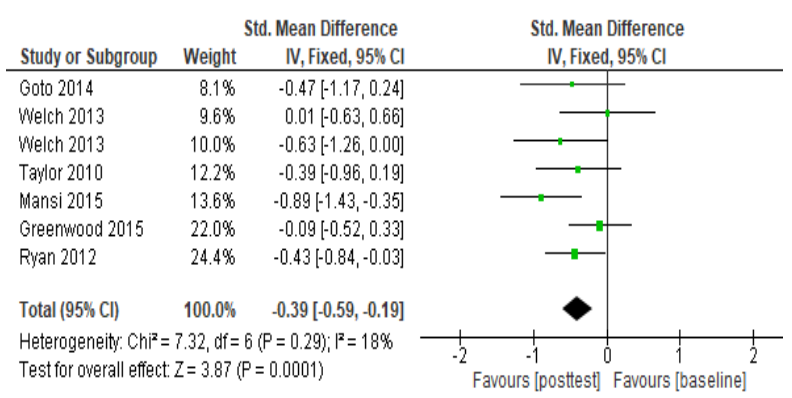

Figure 7. Forest plot showing self-efficacy effect size with $95 \% \mathrm{Cl}$ for each intervention

\section{Discussion}

The meta-analysis of ITSM intervention on weight management and physical activity shows a small but significant effect on body weight change and waist circumference ( $g=0.31$ and 0.3 ), and a significant but negligible effect on BMI $(g=0.15)$. The effect on physical activity is measure-dependent: we found a significant moderate effect on time-based outcomes ( $g=0.63)$, but a small effect on step-based outcomes $(g=0.34)$.

The post-hoc analysis of self-efficacy found a consistent small-to-moderate effect size. The role of this psychological construct is in line with cognitivebehavioral therapy that emphasizes the role of 
cognition in learned behaviors and behavior changes [29]. Since the current set of studies does not have enough information to conduct further analysis of the impact of self-efficacy on weight and physical activity outcomes, future research could formally examine the mediating role of self-efficacy.

Future research could also examine mechanisms in addition to self-efficacy - which mediate the effect of ITSM on health outcomes. Such mechanisms may include perceived engagement, motivation, perceived social support [34] [35] [36]. Finding these mediators may improve our theoretical explanations which would be useful in developing more effective ITSM interventions. In terms of study heterogeneity, a greater variability in effect size estimates has been observed for physical activity studies, indicating potentially non-comparability of the interventions and limited generalizability of the research findings.

However, these heterogeneity issues suggest opportunities for future research. For example, further subgroup analysis and moderator analysis can be conducted to explain such heterogeneity. Table 1 can be a starting point where participant characteristics and intervention characteristics can be used as grouping criteria. Since the intervention design varied significantly, future research could examine how combinations of various intervention components (e.g., such as ITSM paired with goal setting techniques, education sessions, or feedback) influence outcomes.

Another part of the intervention which may explain the issues with heterogeneity is the IT itself. The rapid evolution of technology increases convenience in data recording and also changes the way patients self-manage their chronic conditions and lifestyles. Thus, it is important to investigate the impacts of evolving IT-specific characteristics on both the IT-user interaction and ultimate health outcomes. Interestingly, in our current sample of studies, newer generation technologies such as smart wearables did not outperform older generation tools such as PDAs. However, study design varied significantly, and future studies could more directly investigate how evolving technologies influence ITuser interaction and health outcomes.

Due to the emerging nature of ITSM techniques, the current number of studies is fairly limited, and it is difficult to conduct meaningful subgroup analysis, which is a limitation of current study. Another limitation is the assessment of bias. Future research should formally assess various risks of bias such as allocation concealment, detection bias, and attrition bias to address the quality of evidence. Such bias affects the practical significance and robustness of the conclusions that can be drawn from metaanalyses.

In summary, this study takes a broad and systematic screening approach to include a relatively comprehensive list of recent studies examining ITSM interventions. It updates the previous meta-analysis by incorporating a broader scope of participants and time. The focus on IT-based intervention provides an initial assessment of recent healthcare delivery practices. Our results provide evidence of the effectiveness of ITSM in weight management; however, the effect on physical activity is less consistent, especially for step-based outcomes. Since ITSM tools are becoming more diverse nowadays, and more types of measurements are available for patients to self-manage various aspects of their lives, future research can go beyond weight and physical activity outcomes to investigate a broader range of disease and SM behaviors such as diet, sleeping pattern, smoking, and medication. Furthermore, additional emphasis can be put on opening the black box of IT (e.g. IT capabilities that afford certain therapy goals) and IT usage patterns, and synthesizing their effects.

\section{References}

[1] The top 10 causes of death. World Health Organization, 2018. http://www.who.int/news-room/fact-sheets/detail/thetop-10-causes-of-death.

[2] Fine, L. J., Philogene, G. S., Gramling, R., Coups, E. J. and Sinha, S. Prevalence of multiple chronic disease risk factors. American Journal of Preventive Medicine, 27, 2 (2004), 18-24.

[3] Must, A., Spadano, J., Coakley, E. H., Field, A. E., Colditz, G. and Dietz, W. H. The disease burden associated with overweight and obesity. Journal of the American Medical Association, 282, 16 (1999), 1523-1529.

[4] Owen, N., Salmon, J., Koohsari, M. J., Turrell, G. and Giles-Corti, B. Sedentary behaviour and health: mapping environmental and social contexts to underpin chronic disease prevention. British Journal of Sports Medicine, 48, 3 (2014), 174-177.

[5] Hillestad, R., Bigelow, J., Bower, A., Girosi, F., Meili, R., Scoville, R. and Taylor, R. Can electronic medical record systems transform health care? Potential health benefits, savings, and costs. Health affairs, 24, 5 (2005), 1103-1117.

[6] Kennedy, C. M., Powell, J., Payne, T. H., Ainsworth, J., Boyd, A. and Buchan, I. Active assistance technology for health-related behavior change: An interdisciplinary review. Journal of Medical Internet Research, 14, 3 (MayJun 2012), 138-157.

[7] Hordern, A., Georgiou, A., Whetton, S. and Prgomet, M. Consumer e-health: an overview of research evidence and implications for future policy. Health Information Management Journal, 40, 2 (2011), 6-14. 
[8] Lorig, K. Chronic disease self-management: a model for tertiary prevention. American Behavioral Scientist, 39, 6 (1996), 676-683.

[9] Paré, G., Leaver, C. and Bourget, C. Diffusion of the Digital Health Self-Tracking Movement in Canada: Results of a National Survey. Journal of Medical Internet Research, 20, 5 (2018).

[10] Wilde, M. H. and Garvin, S. A concept analysis of self-monitoring. Journal of Advanced Nursing, 57, 3 (2007), 339-350.

[11] Song, M. and Lipman, T. H. Concept analysis: Selfmonitoring in type 2 diabetes mellitus. International Journal of Nursing Studies, 45, 11 (Nov 2008), 1700-1710.

[12] Li, I., Dey, A. and Forlizzi, J. A stage-based model of personal informatics systems. In Proceedings of the Proceedings of the SIGCHI Conference on Human Factors in Computing Systems (Atlanta, Georgia, USA, 2010). ACM.

[13] Gonzalez, J. S., Peyrot, M., McCarl, L. A., Collins, E. M., Serpa, L., Mimiaga, M. J. and Safren, S. A. Depression and diabetes treatment nonadherence: a meta-analysis. Diabetes care, 31, 12 (2008), 2398-2403.

[14] van Dulmen, S., Sluijs, E., van Dijk, L., de Ridder, D., Heerdink, R. and Bensing, J. Patient adherence to medical treatment: a review of reviews. BMC health services research, 7, 1 (2007), 55.

[15] Burke, L. E., Wang, J. and Sevick, M. A. SelfMonitoring in Weight Loss: A Systematic Review of the Literature. Journal of the American Dietetic Association, 111, 1 (Jan 2011), 92-102.

[16] George, E. S., Kolt, G. S., Duncan, M. J., Caperchione, C. M., Mummery, W. K., Vandelanotte, C., Taylor, P. and Noakes, M. A Review of the Effectiveness of Physical Activity Interventions for Adult Males. Sports Medicine, 42, 4 (2012), 281-300.

[17] Greaves, C. J., Sheppard, K. E., Abraham, C., Hardeman, W., Roden, M., Evans, P. H. and Schwarz, P. Systematic review of reviews of intervention components associated with increased effectiveness in dietary and physical activity interventions. BMC Public Health, 11, 1 (2011), 119-130.

[18] Olander, E. K., Fletcher, H., Williams, S., Atkinson, L., Turner, A. and French, D. P. What are the most effective techniques in changing obese individuals' physical activity self-efficacy and behaviour: a systematic review and meta-analysis. International Journal of Behavioral Nutrition \& Physical Activity, 10 (2013), 29-43.

[19] van den Berg, M. H., Schoones, J. W. and Vlieland, T. P. Internet-based physical activity interventions: A systematic review of the literature. Journal of Medical Internet Research, 9, 3 (2007), p71-p86.

[20] Higgins JPT, Green S (editors). Cochrane Handbook for Systematic Reviews of Interventions Version 5.1.0 [updated March 2011]. The Cochrane Collaboration, 2011. Available from www.handbook.cochrane.org.

[21] McAuley, E. and Blissmer, B. Self-efficacy determinants and consequences of physical activity. Exercice Sport Science Review, 28, 2 (2000), 85-88.

[22] Linde, J. A., Rothman, A. J., Baldwin, A. S. and Jeffery, R. W. The impact of self-efficacy on behavior change and weight change among overweight participants in a weight loss trial. Health Psychology, 25, 3 (2006), 282. [23] Abraham, C. and Michie, S. A Taxonomy of Behavior Change Techniques Used in Interventions. Health Psychology, 27, 3 (2008), 379-387.

[24] Jacobson, N. S., Dobson, K. S. and Truax, P. A. A component analysis of cognitive-behavioral treatment for depression. Journal of Consulting and Clinical Psychology, 64 (1996), 295-304.

[25] Webster, J. and Watson, R. Analyzing the Past to Prepare for the Future: Writing a Literature Review. MIS Quarterly, 26, 2 (2002), xiii-xxiii.

[26] Hedges, L. V. Distribution theory for Glass's estimator of effect size and related estimators. Journal of Educational Statistics, 6, 2 (1981), 107-128.

[27] Cohen, J. A power primer. Psychological bulletin, 112, 1 (1992), 155-159.

[28] Egger, M., Smith, G. D., Schneider, M. and Minder, C. Bias in meta-analysis detected by a simple, graphical test. BMJ, 315, 7109 (1997), 629-634.

[29] Rothbaum, B. O., Meadows, E. A., Resick, P. and Foy, D. W. Cognitive-behavioral therapy (2000).

[30] Allen, J. K., Stephens, J., Dennison Himmelfarb, C. R., Stewart, K. J. and Hauck, S. Randomized Controlled Pilot Study Testing Use of Smartphone Technology for Obesity Treatment. Journal of Obesity, 2013 (2013), 1-7.

[31] Carter, M. C., Burley, V. J., Nykjaer, C. and Cade, J. E. Adherence to a smartphone application for weight loss compared to website and paper diary: Pilot randomized controlled trial. Journal of Medical Internet Research, 15, 4 (Apr 2013), 56-72.

[32] Durlak, J. A. How to Select, Calculate, and Interpret Effect Sizes. Journal of Pediatric Psychology, 34, 9 (2009), 917-928.

[33] Lipsey, M. W. and Wilson, D. B. Practical metaanalysis. Sage Publications, Inc, 2001.

[34] Anderson, K., Burford, O. and Emmerton, L. Mobile Health Apps to Facilitate Self-Care: A Qualitative Study of User Experiences. PLoS ONE, 11, 5 (2016), 1-21.

[35] Morgan, P., Scott, H., Young, M., Plotnikoff, R., Collins, C. and Callister, R. Associations between program outcomes and adherence to Social Cognitive Theory tasks: Process evaluation of the SHED-IT community weight loss trial for men. International Journal of Behavioral Nutrition \& Physical Activity, 11 (2014), 1-28.

[36] Taylor, A. H., Everson-Hock, E. S. and Ussher, M. Integrating the promotion of physical activity within a smoking cessation programme: Findings from collaborative action research in UK Stop Smoking Services. BMC Health Services Research, 10 (2010), 317-327.

\section{Included studies}

Acharya, S. D., Elci, O. U., Sereika, S. M., Styn, M. A., and Burke, L. E. 2011. Using a Personal Digital Assistant for Self-Monitoring Influences Diet Quality in Comparison to a Standard Paper Record among Overweight/Obese Adults, Journal of the American Dietetic Association (111:4), pp. 583-588.

Akers, J. D., Cornett, R. A., Savla, J. S., Davy, K. P., and Davy, B. M. 2012. Daily Self-Monitoring of Body Weight, 
Step Count, Fruit/Vegetable Intake, and Water Consumption: A Feasible and Effective Long-Term Weight Loss Maintenance Approach, Journal of the Academy of Nutrition and Dietetics (112:5), pp. 685-692.

Allen, J. K., Stephens, J., Dennison Himmelfarb, C. R., Stewart, K. J., and Hauck, S. 2013. Randomized Controlled Pilot Study Testing Use of Smartphone Technology for Obesity Treatment, Journal of Obesity (2013), pp. 1-7.

Butryn, M. L., Arigo, D., Raggio, G. A., Colasanti, M., and Forman, E. M. 2016. Enhancing Physical Activity Promotion in Midlife Women with Technology-Based SelfMonitoring and Social Connectivity: A Pilot Study, Journal of Health Psychology (21:8), pp. 1548-1555.

Cadmus-Bertram, L., Marcus, B. H., Patterson, R. E., Parker, B. A., and Morey, B. L. 2015. Use of the Fitbit to Measure Adherence to a Physical Activity Intervention among Overweight or Obese, Postmenopausal Women: Self-Monitoring Trajectory During 16 Weeks, Jmir Mhealth and Uhealth (3:4), pp. 82-88.

Cadmus-Bertram, L., Wang, J. B., ... and Pierce, J. P. 2013. Web-Based Self-Monitoring for Weight Loss among Overweight/Obese Women at Increased Risk for Breast Cancer: The Help Pilot Study, Psycho-Oncology (22:8), pp. $1821-1828$.

Carter, M. C., Burley, V. J., Nykjaer, C., and Cade, J. E. 2013. Adherence to a Smartphone Application for Weight Loss Compared to Website and Paper Diary: Pilot Randomized Controlled Trial, Journal of Medical Internet Research (15:4), pp. 56-72.

Chambliss, H. O., Huber, R. C., ... and Wilkinson, W. J. 2011. Computerized Self-Monitoring and TechnologyAssisted Feedback for Weight Loss with and without an Enhanced Behavioral Component, Patient Education and Counseling (85:3), pp. 375-382.

Dennison, L., Morrison, L., ... and Yardley, L. 2014. Does Brief Telephone Support Improve Engagement with a Web-Based Weight Management Intervention? Randomized Controlled Trial, Journal of Medical Internet Research (16:3), pp. 130-144.

Goto, M., Takedani, H., ... and Nitta, O. 2014. SelfMonitoring Has Potential for Home Exercise Programmes in Patients with Haemophilia, Haemophilia (20:2), pp. e121-e127.

Greenwood, D. A., Blozis, S. A., ... and Quinn, C. C. 2015. Overcoming Clinical Inertia: A Randomized Clinical Trial of a Telehealth Remote Monitoring Intervention Using Paired Glucose Testing in Adults with Type 2 Diabetes, Journal of Medical Internet Research (17:7), e178.

Izawa, K. P., Watanabe, S., Oka, K., Osada, N., and Omiya, K. 2006. Effect of Self-Monitoring Approach During Cardiac Rehabilitation on Exercise Maintenance, SelfEfficacy, and Physical Activity over a 1-Year Period after Myocarcial Infarction, Japanese Journal of Physical Fitness and Sports Medicine (55), pp. 113-118.
Jones, M., Lynch, K. T., ... and Taylor, C. 2014. Healthy Weight Regulation and Eating Disorder Prevention in High School Students: A Universal and Targeted Web-Based Intervention, Journal of Medical Internet Research (16:2), pp. 28-39.

King, A. C., Hekler, E. B., ... and Cirimele, J. 2013. Harnessing Different Motivational Frames Via Mobile Phones to Promote Daily Physical Activity and Reduce Sedentary Behavior in Aging Adults, PLoS ONE (8:4), pp. 1-8.

Kirwan, M., Duncan, M. J., Vandelanotte, C., and Mummery, W. 2012. Using Smartphone Technology to Monitor Physical Activity in the 10,000 Steps Program: A Matched Case-Control Trial, Journal of Medical Internet Research (14:2), pp. 176-185.

Lara, J., O’Brien, N., Godfrey, A., Heaven, B., Evans, E. H., Lloyd, S., Moffatt, S., Moynihan, P. J., Meyer, T. D., Rochester, L., Sniehotta, F. F., White, M., and Mathers, J. C. 2016. Pilot Randomised Controlled Trial of a WebBased Intervention to Promote Healthy Eating, Physical Activity and Meaningful Social Connections Compared with Usual Care Control in People of Retirement Age Recruited from Workplaces, PLOS ONE (11:7), pp. 1-17.

Lin, J. J., Mamykina, L., ... and Strub, H. B. 2006. Fish'n'steps: Encouraging Physical Activity with an Interactive Computer Game, in: Proceedings of the 8th international conference on Ubiquitous Computing. Orange County, CA: Springer-Verlag, pp. 261-278.

Maher, C., Ferguson, M., Vandelanotte, C., Plotnikoff, R., De Bourdeaudhuij, I., Thomas, S., Nelson-Field, K., and Olds, T. 2015. A Web-Based, Social Networking Physical Activity Intervention for Insufficiently Active Adults Delivered Via Facebook App: Randomized Controlled Trial, Journal of Medical Internet Research (17:7), e174.

Mansi, S., Milosavljevic, S., ... and Baxter, D. G. 2015. Investigating the Effect of a 3-Month Workplace-Based Pedometer-Driven Walking Programme on Health-Related Quality of Life in Meat Processing Workers: A Feasibility Study within a Randomized Controlled Trial, BMC Public Health (15:1), pp. 410-421.

Nicklas, B. J., Gaukstern, J. E., Beavers, K. M., Newman, J. C., Leng, X. Y., and Rejeski, W. J. 2014. SelfMonitoring of Spontaneous Physical Activity and Sedentary Behavior to Prevent Weight Regain in Older Adults, Obesity (22:6), pp. 1406-1412.

Ruotsalainen, H., Kyngäs, H., ... and Kääriäinen, M. 2015. Effectiveness of Facebook-Delivered Lifestyle Counselling and Physical Activity Self-Monitoring on Physical Activity and Body Mass Index in Overweight and Obese Adolescents: A Randomized Controlled Trial, Nursing Research \& Practice (2015), pp. 1-14.

Ryan, D., Price, D., ... and Pinnock, H. 2012. Clinical and Cost Effectiveness of Mobile Phone Supported Self Monitoring of Asthma: Multicentre Randomised Controlled Trial, British Medical Journal (344), e1756. 
Schroder, K. E. E. 2011. Computer-Assisted Dieting: Effects of a Randomized Nutrition Intervention, American Journal of Health Behavior (35:2), pp. 175-188.

Shuger, S. L., Barry, V. W., ... and Blair, S. N. 2011. Electronic Feedback in a Diet- and Physical Activity-Based Lifestyle Intervention for Weight Loss: A Randomized Controlled Trial, International Journal of Behavioral Nutrition \& Physical Activity (8), pp. 41-48.

Steinberg, D. M., Levine, E. L., Askew, S., Foley, P., and Bennett, G. G. 2013. Daily Text Messaging for Weight Control among Racial and Ethnic Minority Women: Randomized Controlled Pilot Study, Journal of Medical Internet Research (15:11), pp. 67-77.

Taylor, A. H., Everson-Hock, E. S., and Ussher, M. 2010. Integrating the Promotion of Physical Activity within a Smoking Cessation Programme: Findings from Collaborative Action Research in Uk Stop Smoking Services, BMC Health Services Research (10), pp. 317327.
Thomas, J. G., Leahey, T. M., and Wing, R. R. 2015. An Automated Internet Behavioral Weight-Loss Program by Physician Referral: A Randomized Controlled Trial, Diabetes Care (38:1), pp. 9-15.

Turner-McGrievy, G. M., Beets, M. W., ... and Tate, D. F. 2013. Comparison of Traditional Versus Mobile App SelfMonitoring of Physical Activity and Dietary Intake among Overweight Adults Participating in an Mhealth Weight Loss Program, Journal of the American Medical Informatics Association (20:3), pp. 513-518.

Welch, J. L., Astroth, K. S., ... and Scott, L. L. 2013. Using a Mobile Application to Self-Monitor Diet and Fluid Intake among Adults Receiving Hemodialysis, Research in Nursing \& Health (36:3), pp. 284-298.

Wharton, C. M., Johnston, C. S., Cunningham, B. K., and Sterner, D. 2014. Dietary Self-Monitoring, but Not Dietary Quality, Improves with Use of Smartphone App Technology in an 8-Week Weight Loss Trial, Journal of Nutrition Education and Behavior (46:5), pp. 440-444.

\section{Appendix A. Funnel plot of standard error by standard mean difference for the outcomes}

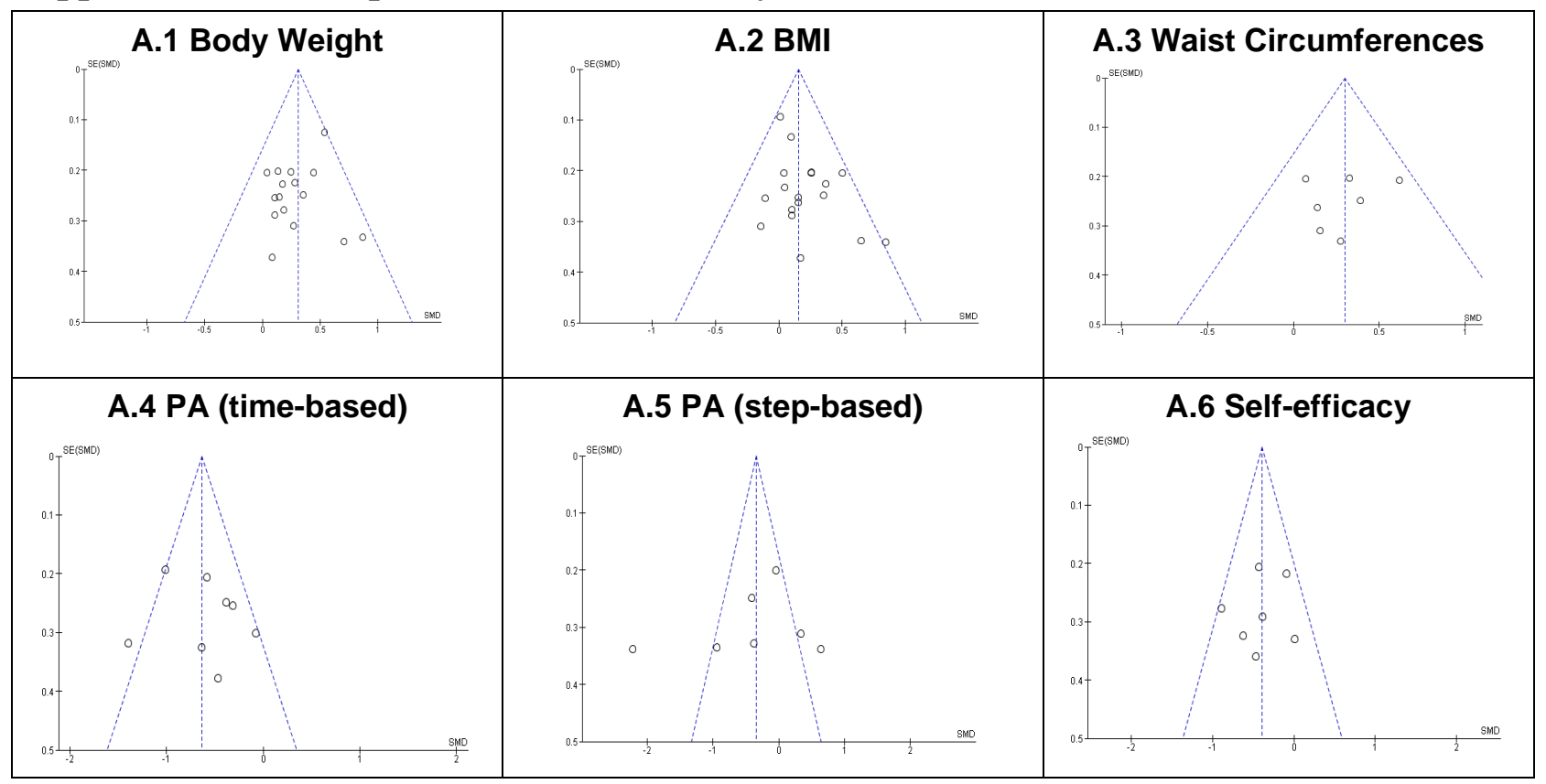

